\title{
Sustainable personal transport
}

\section{Lowson}

\section{S. Bowers, Directorate of Transportation and Technical Services, Daventry District Council}

The paper outlines with some thoroughness the potential benefits of the personal public transport concept, as embodied in the ULTra system. However, it inevitably gives rise to a number of questions, as follows.

(a) It is stated that ULTra offers a solution suitable for cities with populations below 2 million. Have studies also indicated the size of urban area below which a system is unlikely to be viable?

(b) The system is stated to meet the sustainability criteria of $1.8 \mathrm{~kW} / \mathrm{h}$, this being based on vehicle consumption of $2 \cdot 0 \mathrm{~kW} / \mathrm{h}$ with an average occupancy of $1 \cdot 6$, which amounts to $1.3 \mathrm{~kW} / \mathrm{h}$ per person. While this would be a major improvement over other modes, it is not clear that the formal sustainability criteria would be met, because the calculation does not appear to allow for energy consumed in the movement of empty vehicles. Is this the case?

(c) What consideration has been given to the risk of unauthorised access or vandalism on the guideway? The experience of rail, where unauthorised access carries much higher personal risks for the intruder than would be the case in an ULTra system, indicates that this could be a considerable problem.

(d) On a related point, has consideration been given to the effect of birds on the guideway? Particularly, is the system designed to stop automatically if obstructions are detected?

(e) Finally, and perhaps more speculatively, given the low loads imposed by the system, has any consideration been given to the use of advanced composites to reduce the cost and/or visual intrusion of ULTra guideways?
Authors' reply

I would like to thank Mr. Bowers for his complimentary remarks and for his questions. In answer to these the following comments are given.

(a) The minimum viable scale of an ULTra system depends strongly on the nature of the application. We are currently examining a system to link a community of about 2000 people to the city centre. This looks very practicable. Shopping centres and leisure complexes offer other opportunities.

(b) Simulations indicate that typical empty vehicle usage requires an overhead factor of about $1 \cdot 4$. A $2 \mathrm{~kW}$ power usage combined with the projected 1.6 passenger load gives an average usage of $1.75 \mathrm{~kW}$, which meets the sustainability criterion.

(c) Misuse of the system is the dominant issue in our safety studies. We believe that the smart card ticketing system will minimise misuse of the vehicles. The track is fully segregated, either by elevation or by fencing at-grade, and extensively covered by CCTV. A number of technical features are also incorporated in the system to reduce the potential consequences of vandalism. However, this will remain a major problem. Operational experience is required to determine most significant issues and best response.

(d) The track has been designed to provide no nesting opportunities for birds. Automatic devices will be set not to respond to birds.

(e) The design of the system has been driven by minimum cost. Advanced composites do provide a lighter solution, but the stiffness required demands carbon composites, which are significantly more expensive than steel. There is a stronger case for advanced composites for some of the longest spans. 